WSRC-STI-2006-00332, Rev. 0

Key Words:

Environment

Remediation

Retention:

Permanent

\title{
Advancement of Nucleic Acid-Based Tools for Monitoring In Situ Reductive Dechlorination
}

(A Research Study of the Monitored Natural Attenuation/Enhanced Attenuation for Chlorinated Solvents Technology Alternative Project)

November 17, 2006

Washington Savannah River Company
Savannah River Site
Aiken, SC 29808
$\begin{aligned} & \text { Prepared for the U.S. Department of Energy } \\ & \text { Under Contract Number DEAC09-96- } \\ & \text { SR18500 }\end{aligned}$


WSRC-STI-2006-00332, Rev. 0

\section{DISCLAIMER}

Preparation of this report was coordinated by Washington Savannah River Company (WSRC) for the United States Department of Energy (US DOE) under Contract No. DE-AC09-96SR18500. Extensive effort was made by the authors to assure the accuracy of the contents and interpretation. However, the USDOE nor WSRC, nor any of their employees makes any warranty, expressed or implied, or assumes any legal liability or responsibility for accuracy, completeness, or usefulness, of any information, apparatus, or product or process disclosed herein or represents that its use will not infringe privately owned rights. Reference herein to any specific commercial product, process, or service by trade name, trademark, name, manufacturer or otherwise does not necessarily constitute or imply endorsement, recommendation, or favoring of same by Washington Savannah River Company or by the United States Government or any agency thereof. The views and opinions of the authors expressed herein do not necessarily state or reflect those of the United States Government or any agency thereof.

Printed in the United States of America

Prepared For

U.S. Department of Energy 
WSRC-STI-2006-00332, Rev. 0

November 17, 2006

Page I-iii of I-vii

Key Words:

Environment

Remediation

Retention:

Permanent

\section{Advancement of Nucleic Acid-Based Tools for Monitoring In Situ Reductive Dechlorination}

(A Research Study of the Monitored Natural Attenuation/Enhanced Attenuation for Chlorinated Solvents Technology Alternative Project)

Contributing Researchers:

Elizabeth Edwards, University of Toronto

Frank Löffler, Georgia Institute of Technology

Introduced by:

Karen Vangelas, SRNL

Brian B. Looney, SRNL

November 17, 2006

$\begin{aligned} & \text { Washington Savannah River Company } \\ & \text { Savannah River Site } \\ & \text { Aiken, SC } 29808\end{aligned}$
$\begin{aligned} & \text { Prepared for the U.S. Department of Energy } \\ & \text { Under Contract Number DEAC09-96- } \\ & \text { SR18500 }\end{aligned}$




\section{Executive Summary}

Regulatory protocols generally recognize that destructive processes are the most effective mechanisms that support natural attenuation of chlorinated solvents. In many cases, these destructive processes will be biological processes and, for chlorinated compounds, will often be reductive processes that occur under anaerobic conditions. The existing EPA guidance (EPA, 1998) provides a list of parameters that provide indirect evidence of reductive dechlorination processes. In an effort to gather direct evidence of these processes, scientists have identified key microorganisms and are currently developing tools to measure the abundance and activity of these organisms in subsurface systems. Drs. Edwards and Löffler are two recognized leaders in this field. The research described herein continues their development efforts to provide a suite of tools to enable direct measures of biological processes related to the reductive dechlorination of TCE and PCE. This study investigated the strengths and weaknesses of the $16 \mathrm{~S}$ rRNA gene-based approach to characterizing the natural attenuation capabilities in samples. The results suggested that an approach based solely on 16S rRNA may not provide sufficient information to document the natural attenuation capabilities in a system because it does not distinguish between strains of organisms that have different biodegradation capabilities. The results of the investigations provided evidence that tools focusing on relevant enzymes for functionally desired characteristics may be useful adjuncts to the 16SrRNA methods.

\section{Introduction}

Over the past three decades, much progress has been made in the remediation of soil and groundwater contaminated by chlorinated solvents. Yet these pervasive contaminants continue to present a significant challenge to the U.S. Department of Energy (DOE), other federal agencies, and other public and private organizations. The physical and chemical properties of chlorinated solvents make it difficult to rapidly reach the low concentrations typically set as regulatory limits. These technical challenges often result in high costs and long remediation time frames. In 2003, the DOE through the Office of Environmental Management funded a science-based technical project that uses the U.S. Environmental Protection Agency's technical protocol (EPA, 1998) and directives (EPA, 1999) on Monitored Natural Attenuation (MNA) as the foundation on which to introduce supporting concepts and new scientific developments that will support remediation of chlorinated solvents based on natural attenuation processes. This project supports the direction in which many site owners want to move to complete the remediation of their site(s), that being to complete the active treatment portion of the remedial effort and transition into MNA.

The overarching objective of the effort was to examine environmental remedies that are based on natural processes remedies such as Monitored Natural Attenuation (MNA) or Enhanced Attenuation (EA). The research program did identify several specific opportunities for advances based on: 1) mass balance as the central framework for attenuation based remedies, 2) scientific 
WSRC-STI-2006-00332, Rev. 0

November 17, 2006

Page I-v of I-vii

advancements and achievements during the past ten years, 3) regulatory and policy development and real-world experience using MNA, and 4) exploration of various ideas for integrating attenuation remedies into a systematic set of "combined remedies" for contaminated sites. These opportunities are summarized herein and are addressed in more detail in referenced project documents and journal articles, as well as in the technical and regulatory documents being developed within the Interstate Technology Regulatory Council.

Three topic areas were identified to facilitate development during this project. Each of these topic areas, 1) mass balance, 2) enhanced attenuation (EA), and 3) innovative characterization and monitoring, was explored in terms of policy, basic and applied research, and the results integrated into a technical approach. Each of these topics is documented in stand alone reports, WSRC-STI-2006-00082, WSRC-STI-2006-00083, and WSRC-STI-2006-00084, respectively. In brief, the mass balance efforts are examining methods and tools to allow a site to be evaluated in terms of a system where the inputs, or loading, are compared to the attenuation and destruction mechanisms and outputs from the system to assess if a plume is growing, stable or shrinking. A key in the mass balance is accounting for the key attenuation processes in the system and determining their rates. EA is an emerging concept that is recognized as a transition step between traditional treatments and MNA. EA facilitates and enables natural attenuation processes to occur in a sustainable manner to allow transition from the primary treatment to MNA. EA technologies are designed to either boost the level of the natural attenuation processes or decrease the loading of contaminants to the system for a period of time sufficient to allow the remedial goals to be met over the long-term. For characterization and monitoring, a phased approach based on documenting the site specific mass balance was developed. Tools and techniques to support the approach included direct measures of the biological processes and various tools to support cost-effective long-term monitoring of systems where the natural attenuation processes are the main treatment remedies. The effort revealed opportunities for integrating attenuation mechanisms into a systematic set of "combined remedies" for contaminated sites.

An important portion of this project was a suite of 14 research studies that supported the development of the three topic areas. A research study could support one or more of these three topic areas, with one area identified as the primary target. There were three funded studies addressing the use of molecular biological tools to support natural attenuation remedial processes. The objective of one of the studies was to develop a molecular probe to detect the JS666 bacteria that is an aerobic bacteria capable of degrading cis-1,2-dichloroethene. The objective of the second study was to examine the use of messenger RNA (mRNA) of Dehalococcoides, a microorganism linked to the anaerobic reductive dechlorination of tetrachloroethene (PCE) and trichloroethene (TCE) and protein measurements to directly measure the rate of biological degradation in "realtime" for use in mass balance modeling. The objective of the third study was to investigate the strengths and weaknesses of the 16S rRNA gene-based approach to characterizing the natural attenuation capabilities in samples containing Dehalococcoides. 
WSRC-STI-2006-00332, Rev. 0

November 17, 2006

Page I-vi of I-vii

The attached report documents the results of the development efforts of molecular tools associated with supporting the third objective above. This study was led by Elizabeth Edwards of the University of Toronto and Frank Löffler of the Georgia Institute of Technology. This work leverages work conducted by this team for other agencies including the Department of Defense, the U.S. Environmental Protection Agency, and the National Science Foundation. This study supports the topic area(s) of mass balance and characterization and monitoring. This study had two phases, with specific objectives for each phase. These objectives were:

Phase 1: a) refine the $16 \mathrm{~S}$ rRNA gene-based approach and define its limitations

b) use a variety of approaches to screen a variety of samples (pure, mixed environmental) with the goal to identify key genes involved in the reductive dechlorination process

c) establish a link between the presence (and expression) of a particular gene (or set of genes) with an observable trait using a variety of approaches

Phase 2: test and validate the nucleic acid-based tool kit designed in Phase 1 using samples from SRS test beds.

The researchers completed the Phase 1 objectives with results in some areas that were more promising than in others. Due to time limitations the researchers did not complete Phase 2 with SRS samples, but did validate the tool kit with samples from other sites with chlorinated solvent contamination.

The Technical Working Group (TWG) of the MNA/EA for Chlorinated Solvents Project identified the development of molecular biological tools (MBTs) as a key technical target for research supporting this project. This is a growing area in the environmental arena. Being able to directly measure a system's ability to degrade a specific contaminant(s) and the sustainability of that ability will be important factors in providing defensible documentation that an attenuation based remedy is a viable option for a contaminated site. Though making significant progress to providing tools to measure these parameters, those researching this area have only scratched the surface. This study by Edwards and Löffler identified key genes involved in the reductive dechlorination process and investigates links between the presence and expression of a particular gene or set of genes with an observable contaminant degradation capability. Their research did document that 16s rRNA has insufficient information to distinguish physiologically important capabilities of different strains. Their research suggested that tools that focus on the relevant enzymes for functionally desired characteristics may be useful and important adjuncts to the $16 \mathrm{~S}$ rRNA measurements.

The TWG in reviewing this work believe that differentiating between the strains of dechlorinating organisms and elucidating the strengths and weaknesses of the metabolic pathways in each is important and useful in developing molecular biological tools to support characterization and monitoring of attenuation based remedies. The results of this work along with ongoing developments in this area will lead to analytical tools that 
WSRC-STI-2006-00332, Rev. 0

November 17, 2006

Page I-vii of I-vii

can be used to assess the current and future metabolic potential of reductive dechlorination processes. Of particular importance will be the ability to assess capacity and monitor sustainability of biological processes. Though the research is promising, there are many challenges remaining before MBTs will be commercially viable as characterization and monitoring tools.

Through ongoing discussions the TWG came to consensus on the use of MBTs. 1. Use MBTs when you need to (i.e., when there are data that you can't explain). If a site works well, one does not need the detail.

2. Consider synergistic use of MBTs and traditional geochemical measurements. For example, select and use appropriate MBTs based on the biogeochemical gradient (within the plume) to document how and why things are different from place to place.

3. Use MBTs to provide biomarkers (or bioindicators) indicating activity - there are also bioindicators or biomarkers that will directly indicate that a process will not work.

\section{References for Introduction}

EPA, 1998. Technical Protocol for Evaluating Natural Attenuation of Chlorinated Solvents in Groundwater, EPA/600/R-98/128. Washington DC. September 1998.

EPA, 1999. Use of Monitored Natural Attenuation at Superfund, RCRA Corrective Action and Underground Storage Tank Sites, OSWER Directive 9200.4-17P. Washington DC. April 21, 1999.

WSRC-STI-2006-00082, 2006. Mass Balance: A Key to Advancing Monitored and Enhanced Attenuation for Chlorinated Solvents. Washington Savannah River Company, Aiken, SC, 29808. June 2006. Available at www.osti.gov.

WSRC-STI-2006-00083, 2006. Enhanced Attenuation: A Reference Guide on Approaches to Increase the Natural Treatment Capacity of a System, Revision 1. Washington Savannah River Company, Aiken, SC, 29808. August 2006. Available at www.osti.gov.

WSRC-STI-2006-00084, 2006. Characterization and Monitoring of Natural Attenuation of Chlorinated Solvents in Ground Water: A Systems Approach, Revision 1. Washington Savannah River Company, Aiken, SC, 29808. August 2006. Available at www.osti.gov. 
WSRC-STI-2006-00332, Rev. 0

November 17, 2006

This page intentionally left blank. 
WSRC-STI-2006-00332, Rev. 0

November 17, 2006

\section{Attachment}

Report on Research Efforts by Drs. Löffler and Edwards to support the MNA/EA for Chlorinated Solvents Technology Alternative Project 
WSRC-STI-2006-00332, Rev. 0

November 17, 2006

This page intentionally left blank. 


\title{
Advancement of Nucleic Acid-Based Tools for Monitoring In Situ Reductive Dechlorination
}

\author{
Elizabeth Edwards and Frank Löffler
}

Final Report - October 2006

\section{Table of Contents}

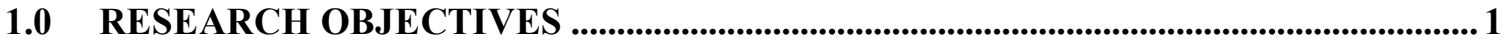

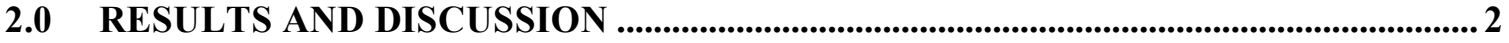

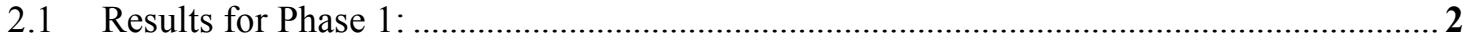

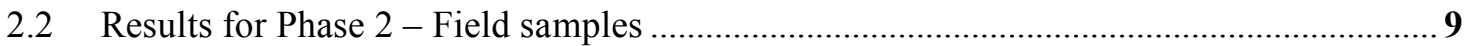

3.0 SUMMARY AND FUTURE WORK................................................................................... 9

4.0 COLLABORATIVE EFFORTS, VISITS TO OTHER LABORATORIES ..................9

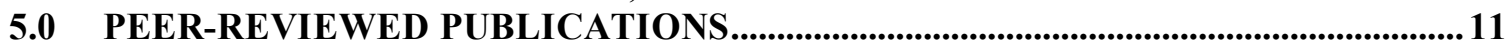

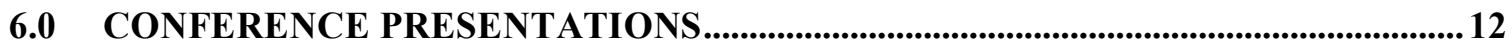

7.0 RELATED PROPOSAL SUBMISSIONS................................................................................ 13

\subsection{RESEARCH OBJECTIVES}

This project addressed the following high priority technical needs for Monitored Natural Attenuation and Enhanced Attenuation (MNA/EA): (i) design of nucleic acid-based site assessment tools, (ii) development of correlations between phylogenetic and functional gene information and chlorinated ethenes reductive dechlorination activity, and (iii) further characterization of chlorinated ethenes-dechlorinating enrichment cultures.

The current state of knowledge allows, at best, short-term predictions on plume development during MNA or implementation of more aggressive treatment(s) including biostimulation and bioaugmentation (EA). Limited data are available on the sustainability of natural attenuation processes, and predictions on whether MNA or EA can be used with confidence for long-term plume control are elusive. Hence, the overall goal of this research effort was to design tools and provide information for guidance protocols for assessment and monitoring of the key microbial populations responsible for chlorinated ethenes removal in plumes undergoing MNA or EA, and to make meaningful predictions on long-term plume development. The availability of rigorously tested tools and protocols will demonstrate whether MNA and EA are viable approaches that can be used with confidence at high-risk DOE waste sites. With the increased knowledge and understanding of the dechlorination process, along with appropriate assessment tools, site managers and regulators will have the means to convincingly argue that MNA and EA are viable, cost effective approaches for plume control and long-lasting risk reduction. This work was conducted in two phases.

The aims of Phase 1 were:

1. to refine the $16 \mathrm{~S}$ rRNA gene-based approach and define its limitations.

2. to design PCR-based approaches that target reductive dehalogenase (RDase) genes implicated in chlorinated ethene reductive dechlorination, and to environmental samples for the presence of RDase genes involved in chlorinated ethene reductive dechlorination. 
3. to establish a link between the presence (and expression) of a particular gene (or set of genes) with an observable trait (e.g., reductive dechlorination of VC to ethene).

4. to explore microarray technology for identification of novel reductive dechlorination biomarker genes.

5. to further characterize chlorinated solvent-dechlorinating enrichment cultures and obtain additional dechlorinating isolates.

The aim of Phase 2 was:

6. to test and validate the nucleic acid-based tools designed in Phase 1 using samples collected at field sites.

\subsection{RESULTS AND DISCUSSION}

\subsection{Phase 1 Results:}

\section{A. Refinement of 16S rRNA gene-based PCR approaches.}

Laboratory and field evidence linked complete reductive dechlorination of chlorinated ethenes to environmentally benign ethene to the presence of Dehalococcoides bacteria. Hence, the focus was on 16S rRNA gene-based approaches to distinguish Dehalococcoides strains capable of efficient vinyl chloride (VC) to ethene formation from those Dehalococcoides strains that cannot dechlorinate chlorinated ethenes or co-metabolize $\mathrm{VC}$, which is a slow and incomplete reaction.

Based on 16S rRNA gene sequence differences, Dehalococcoides group was originally divided into the Cornell, Victoria and Pinellas groups. Dehalococcoides ethenogenes strain 195 belongs to the Cornell group and co-metabolizes VC to ethene. In contrast, Dehalococcoides sp. strain BAV1, a member of the Pinellas group, grew with VC as electron acceptor and produced ethene more efficiently. Hence, we explored if 16S rRNA gene-based analysis is useful to distinguish Dehalococcoides strains belonging to different groups and whether or not this grouping reflects physiological capabilities (i.e., reductive dechlorination of chlorinated ethenes). The alignment of 16S rRNA gene sequences from the few Dehalococcoides isolates and environmental clone sequences available in GenBank allowed us to design 16S rRNA gene-targeted primers that distinguish members of the Cornell, Victoria and Pinellas groups. These tools distinguished the VCrespiring strain BAV1 from the strain 195, which cannot grow with VC. Three other Pinellas isolates, strain CBDB1, strain FL2 and strain GT share identical 16S rRNA gene sequences, yet each strain has distinct dechlorination capabilities. Strain CBDB1 fails to dechlorinate chlorinated ethenes, strain FL2 grows with trichloroethene (TCE) and cis-1,2-dichloroethene (cis-DCE) as electron acceptors but dechlorinates VC only co-metabolically, whereas strain GT respires TCE, cis-DCE and VC and efficiently produces ethene. These finding demonstrate that the Dehalococcoides 16S rRNA gene carries insufficient information to infer physiological properties (i.e., reductive dechlorination activity).

Nevertheless, the 16S rRNA gene-based approach is a valuable tool to detect Dehalococcoides bacteria in environmental samples. A first round PCE with bacterial 16S rRNA gene-targeted primers followed by a second round of PCR with Dehalococcoides 16S rRNA gene-targeted primers (i.e., nested PCR) allows detection of Dehalococcoides organisms in environmental samples with unsurpassed sensitivity. This approach allows the detection of as few as 100 cells per liter of groundwater. We further designed, optimized and validated a quantitative real-time PCR approach to enumerate Dehalococcoides 16S rRNA genes in laboratory and environmental samples. Despite its limitations to distinguish individual Dehalococcoides strains, the 16S rRNA gene approach provides relevant information on Dehalococcoides presence and abundance. To gain an understanding of the relative abundance of Dehalococcoides organisms in a given sample, we designed qPCR protocols to 
enumerate total Bacteria and Archaea. The combined application of these quantitative tools provides information on the relative abundance of Dehalococcoides 16S rRNA genes in mixed cultures or environmental samples. Despite the limited resolution of the 16S rRNA gene-based approach, these tools are useful for monitoring the Dehalococcoides community at MNA/EA sites over temporal and spatial scales. The application of these 16S rRNA gene-based approaches have been optimized and validated with field samples, and protocols for their application have been published (see section 5.0).

\section{B. RDase-targeted PCR approaches.}

To overcome the limitations of the 16S rRNA gene-based approach, we designed PCR approaches to specifically detect the $t c e A, v c r A$ and $b v c A$ genes implicated in chlorinated ethene reductive dechlorination. The tceA gene was detected in Dehalococcoides ethenogenes strain 195 and Dehalococcoides sp. strain FL2 and is implicated in TCE-to-ethene-dechlorination. The RDase genes $v c r A$ and $b v c A$ were identified in Dehalococcoides sp. strain VS and strain BAV1, repectively, and are involved in VC-to-ethene dechlorination. qPCR approaches using linear hybridization (i.e., TaqMan) probe reporter systems were designed to detect and quantify the $t c e A, v c r A$ and $b v c A$ genes in laboratory cultures and environmental samples. This approach was productive and useful to monitor individual RDase genes, and hence specific Dehalococcoides strains in environmental samples.

The combined application of 16S rRNA gene- and RDase gene-targeted approaches provides additional information about the chlorinated ethenes-dechlorinating Dehalococcoides population. Figure 1 shows the relative abundance of Dehalococcoides bacteria following bioaugmentation with BioDechlor INOCULUM, a commercial PCE-to-ethene dechlorinating culture containing Dehalococcoides sp. strain FL2 (possessing tceA), strain BAV1 (possessing $b v c A$ ) and strain GT (possessing $v c r A$ ) at a site where no native Dehalococcoides had been detected. The Dehalococcoides community was maintained for at least 14 months following inoculation, and the quantitative assessment of the $t c e A, v c r A$ and $b v c A$ RDase genes suggested temporal dynamics of individual Dehalococcoides populations with distinct dechlorination properties.
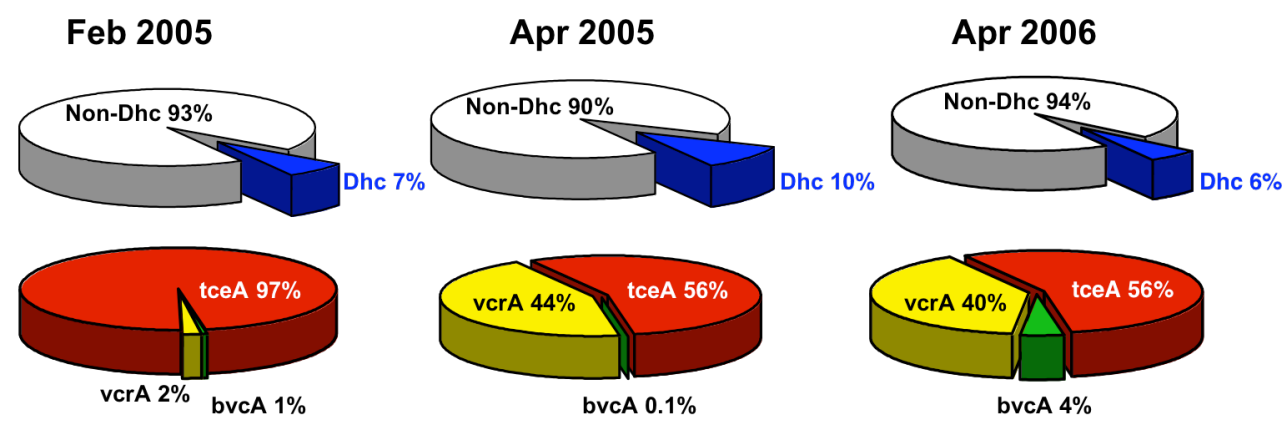

Figure 1: Relative abundance of Dehalococcoides bacteria following bioaugmentation with a PCE-to-ethene dechlorinating culture (BioDechlor INOCULUM) at a site where no native Dehalococcoides had been detected. The bottom part of the figure shows the relative abundances of the $t c e A, v c r A$ and $b v c A$ genes.

\section{Linking RDase gene presence and expression with dechlorination activity.}

Targeting Dehalococcoides 16S rRNA genes and RDase genes implicated in chlorinated ethene reductive dechlorination provides valuable information about their presence and abundance; however, DNA-based tools cannot provide information about gene expression and dechlorinating activity. To 
assess gene expression, different RNA extraction procedures were tested on Dehalococcoides pure cultures and Dehalococcoides-containing mixed cultures. These efforts formed the basis to expand these efforts to RNA extraction from groundwater samples from field sites. A protocol for RNA extraction from groundwater is in its final stages of testing, and we anticipate that the procedures developed will serve as a foundation for the design of standard procedures and protocols for RNA analyses of groundwater samples. Our efforts with pure and mixed cultures demonstrated that the expression of specific RDase genes (e.g., tce $A, v c r A$ and $b v c A$ ) can be detected and quantified using a reverse transcriptase (RT)-qPCR approach.

The applicability of the RT-qPCR approach was tested on groundwater samples collected from a site undergoing EA including biostimulation with lactate and bioaugmentation with BDI.

Groundwater samples (1 L each) were collected for biomass concentration and nucleic acid extraction. The biomass of duplicate groundwater samples was harvested on-site by vacuum filtration, and the membrane filters were flash frozen in liquid nitrogen. Two 1-L bottles were transported on blue ice to the laboratory and stored for 12 hours prior to biomass collection by vacuum filtration. RNA extraction from all samples was performed simultaneously. Figure 2 demonstrates that target $16 \mathrm{~S}$ rRNA and tce $A, v c r A$ and $b v c A$ mRNAs were successfully detected and quantified in groundwater samples collected from the site undergoing EA. DNA was extracted from the same samples and the Dehalococcoides 16S rRNA genes and tce A, vcr $A$ and $b v c A$ genes were quantified.

Figure 2: Quantification of Dehalococcoides $16 \mathrm{~S}$ rRNA and RDase mRNA in total RNA extracts from groundwater (top). Also shown is the quantification of Dehalococcoides 16S rRNA genes and RDase genes (bottom).

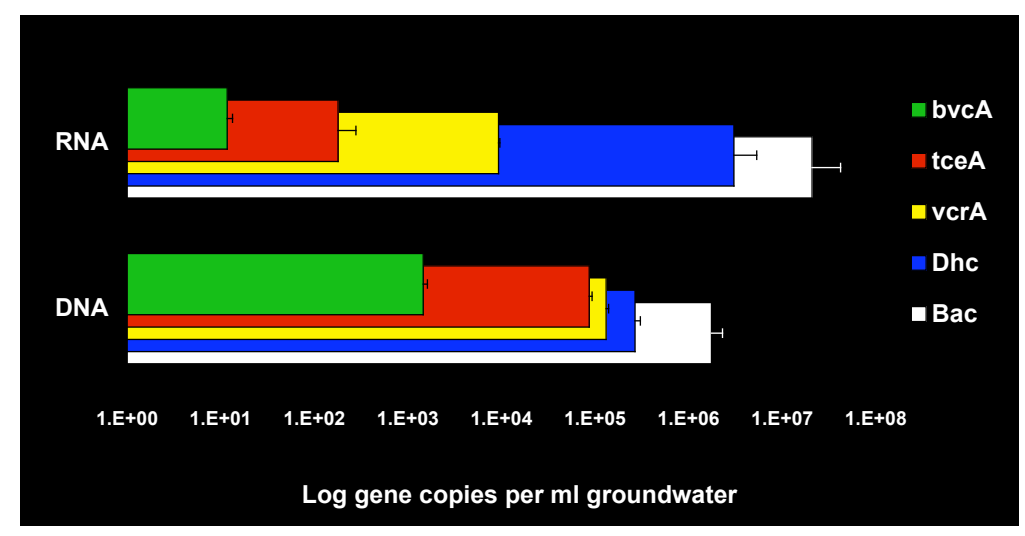

Similar results were obtained with samples that were flash frozen immediately after collection and groundwater samples that were stored at $4^{\circ} \mathrm{C}$ for 24 hours. These preliminary findings suggest that RNA analysis of groundwater samples is feasible, and can provide information on overall Dehalococcoides activity and the expression of specific RDase genes. Further development and optimization is required to advance this approach, and ultimately link target transcript (i.e., mRNA) abundance to activity and provide practitioners with estimates on in-situ dechlorination rates.

PCR primers were also developed for four distinct Dehalobacter-type RDase genes from cultures that dechlorinate 1,2-dichloroethane, 1,1,2-trichloroethane and 1,1,1-trichloroethane (see below). These cultures dechlorinate PCE and TCE to cis-DCE as final product. Transcription of these RDase genes was verified by testing cDNA samples obtained from enrichment cultures exposed to different chlorinated ethanes and ethenes provided as electron acceptors and these genes appear to be specific to the chlorinated ethanes tested. More work is required to determine how effective this screening will be on environmental samples. 


\section{Identification of additional biomarker genes for reductive dechlorination.}

To identify new biomarker genes involved in reductive dechlorination, several approaches were taken, including culture-based experiments and preliminary microarray experiments. Microarray experiments are described below, while the results of culture-based experiments are summarized in section 5 .

Microarray experiments. Two types of microarrays were designed for screening for identification of chlorinated ethene biomarker genes: 1) a shotgun DNA microarray array and 2) a reductive dehalogenase (RDase) array.

The shotgun DNA microarray is made from many random DNA fragments from dechlorinating mixed culture KB-1 spotted onto a glass microscope slide. A small number of prototype arrays were fabricated (printed) with several thousand random DNA fragments from KB-1 to optimize the printing process and array protocols. Using the prototype microarrays, we completed the refinement and optimization of microarray experimental protocols adapted to anaerobic mixed cultures and samples containing FeS and other groundwater constituents. All steps including RNA extraction, reverse transcription, labeling, hybridization and analysis have been optimized. RNA samples extracted from KB-1 cultures during dechlorination of different chlorinated ethenes (i.e., TCE, VC) and 1,2-DCA were compared to RNA extracted from KB-1 cultures grown in the absence of chloroorganic compounds. Analysis of the arrays showed that multiple RDase genes were transcribed simultaneously during dechlorination of a single chlorinated compound, and that some RDase genes were transcribed under all conditions tested. In particular, vcrA was found to be most highly transcribed when KB-1 was grown on vinyl chloride. Reverse transcriptase qPCR experiments confirmed the microarray results (Waller et al. 2005). In addition to genes of known sequence, such as the RDase genes, we also identified several genes of currently unknown function that were upregulated during dechlorination. Some of these up-regulated genes were not from Dehalococcoides, providing an avenue to explore the synergistic relationships between acetogens, methanogens and Dehalococcoides organisms in this culture. More work is required to identify and validate these results.

Unlike the shotgun DNA microarray, the RDase array targets known DNA sequences (i.e., all known RDase genes). As such, it will be useful to explore the diversity and function of these RDase genes, but unknown genes will not be discovered. All RDase gene sequences available in public databases and the numerous Dehalococcoides RDase sequences we have discovered in our laboratories were aligned, compared and combined in an Excel spreadsheet. Also included were hydrogenase, regulatory and housekeeping genes detected in the available Dehalococcoides genomes. The final version of the spreadsheet combines all unique sequences and provides detailed information on the origin of each sequence (e.g., which culture, culture conditions, dechlorinating activity, etc.). In total, we have so far identified 302 unique RDase gene sequences, 104 hydrogenase gene sequences and 58 "control" gene sequences (e.g., select housekeeping genes and 16S rRNA genes). The probe design was completed and three distinct oligonucleotides were synthesized per target gene and spotted onto glass slides. Prototypes of the spotted oligoarrays are now available to explore the expression of RDase, hydrogenase and regulatory genes in Dehalococcoides pure culture and Dehalococcoides-containing consortia grown with different chlorinated electron acceptors. 


\section{E. Characterization of chlorinated solvent-dechlorinating cultures and isolation efforts.}

Several PCE-to-ethene-dechlorinating mixed cultures were obtained from contaminated and pristine sites, as well as enrichment cultures capable of dechlorinating 1,2-DCA, 1,1,2-TCA, 1,1,1TCA and 1,1,-DCA were established.

The PCE-to-ethene-dechlorinating enrichment cultures yielded two novel isolates, Dehalococcoides sp. strain GT and Geobacter lovleyi strain SZ. Strain GT is the first Dehalococcoides isolate that grows with TCE, cis-DCE and VC as electron acceptor (Sung et al. 2006a). Cultures of strain GT provided with acetate as the carbon source, hydrogen as the electron donor and TCE as the electron acceptor, reduced TCE to ethene with very little intermediate formation of cis-DCE and VC (Figure 3).

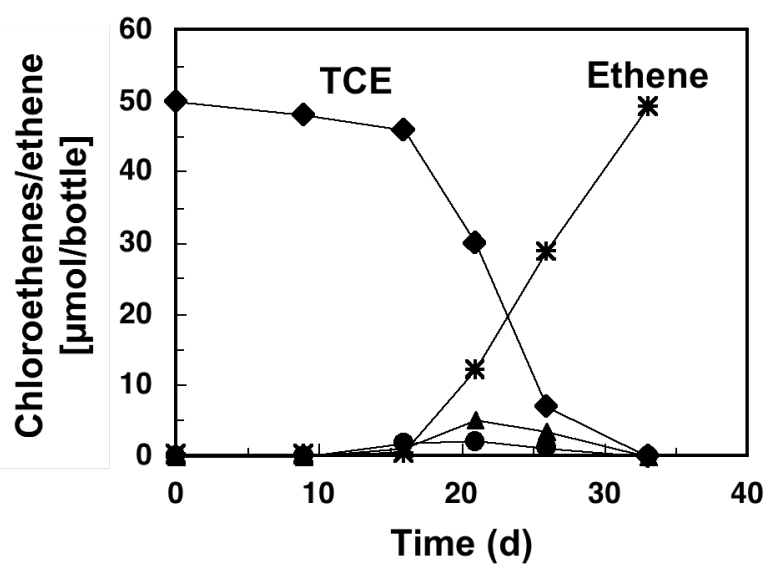

Figure 3: Reductive dechlorination of TCE to ethene by isolate Dehalococcoides sp. strain GT. Note that very little formation of cis-DCE (circles) and VC (triangles) occurred during TCE dechlorination to ethene suggesting that this type of organism may avoid the "cis-DCE and VC stall" often observed at chlorinated ethene contaminated sites.

Another isolate obtained from a PCE-to-ethene-dechlorinating enrichment culture was identified as the first Geobacter species capable of PCE-to-cis-DCE reductive dechlorination. The new isolate was designated Geobacter lovleyi strain SZ (Sung et al. 2006b). Strain SZ also reduced soluble and mobile hexavalent uranium, U(VI), to immobile U(IV) (uraninite). Figure 4 shows that both electron acceptors, PCE and U(VI), were reduced concomitantly indicating that Geobacter lovleyi has promising characteristics for bioremediation applications at mixed waste sites.

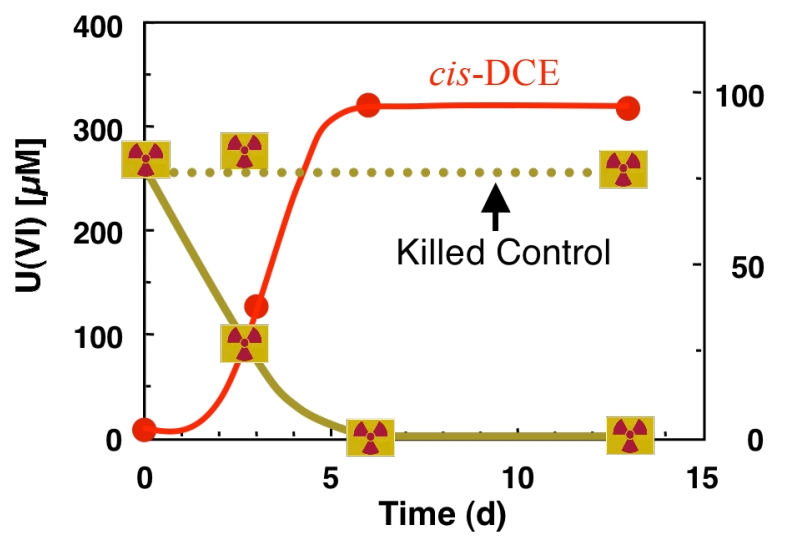

Figure 4: Simultaneous reduction of PCE to cis-DCE and U(VI) to U(IV) by Geobacter lovleyi strain SZ. 
An enrichment culture capable of 1,1,2-TCA and 1,2-DCA transformation was developed from a site contaminated with both chlorinated ethenes and ethanes. This culture was maintained with 1,1,2trichloroethane as electron acceptor and ethanol as electron donor (Figure 5).

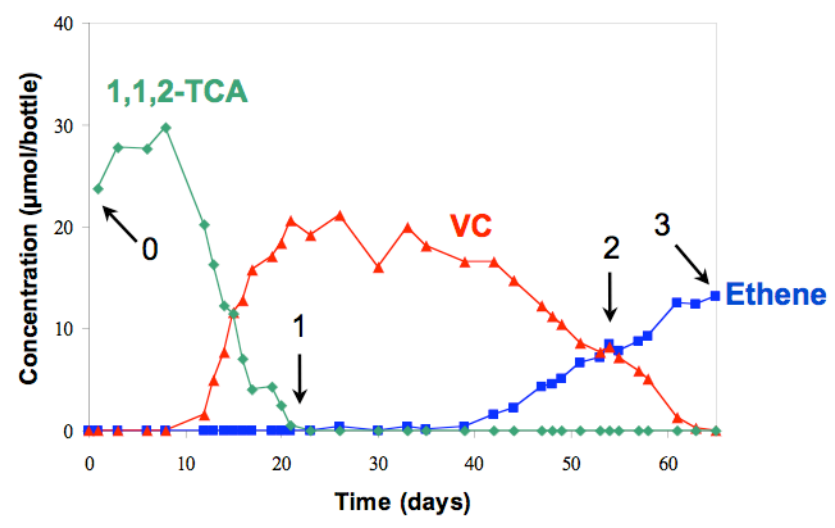

Figure 5: Dechlorination of 1,1,2-TCA in an enrichment culture. DNA was extracted at the time points indicated with arrows $(0$, $1,2,3)$.

This culture was determined to be dominated by Dehalobacter and Dehalococcoides. The growth of Dehalobacter and Dehalococcoides during different steps of dechlorination was determined using qPCR targeting the 16S rRNA gene specific for these two genera. Using this method, we were able to show that Dehalobacter grew only during the dihaloelimination step from 1,1,2-TCA to VC, while Dehalococcoides grew only during the reductive dechlorination of VC to ethene (Figure 6 and Grostern and Edwards, 2006a). These data illustrate that both genera are required for complete dechlorination of 1,1,2-TCA, as summarized schematically in Figure 7.
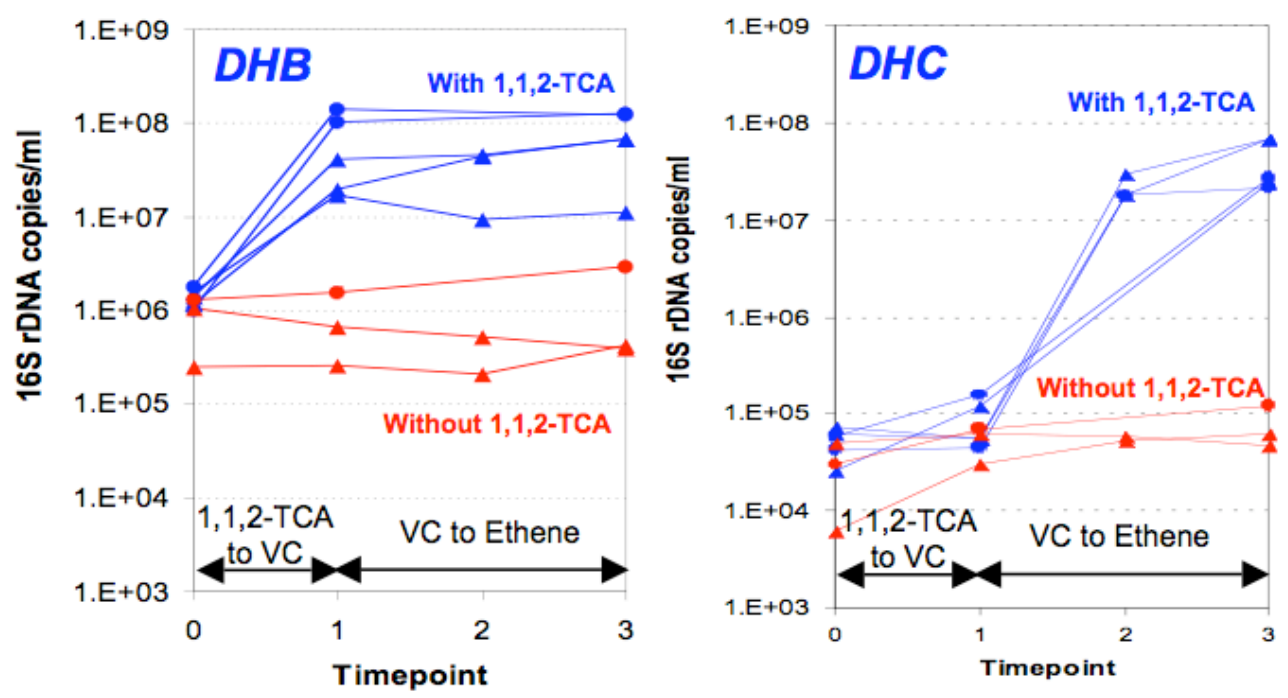

Figure 6: Growth of Dehalobacter $(D H B)$ and Dehalococcoides $(D H C)$ during dechlorination of 1,1,2-TCA via VC to ethene. Each line represents a different culture bottle (blue lines with 1,1,2TCA, red lines without). 
A similar approach was taken with cultures enriched from a 1,1,1-TCA-contaminated site. These enrichment cultures dechlorinate 1,1,1-TCA via 1,1-DCA to chloroethane and no further. It was shown that dechlorination was metabolic, and that one or more organisms belonging to the genus Dehalobacter were responsible for reductive dechlorination of 1,1,1-TCA (Figure 7).
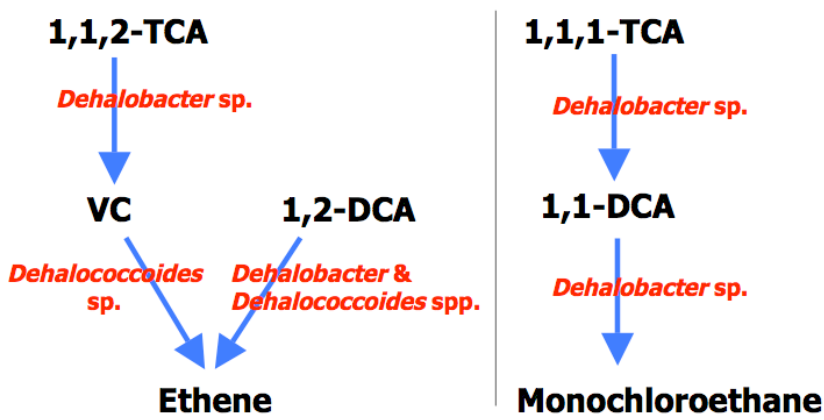

Figure 7: Involvement of Dehalobacter and Dehaloccocoides in dechlorination of chlorinated ethanes (Grostern and Edwards, 2006b)

In addition, given that 1,1,1-TCA is a frequent co-contaminant with TCE and is a known inhibitor of methanogenesis and reductive dechlorination of chlorinated ethenes, we investigated the effects of degradation of mixtures of TCE and 1,1,1-TCA. For example, TCE dechlorination by KB-1 normally proceeds rapidly to ethene. However, when KB-1 is incubated with both TCE and 1,1,1TCA, dechlorination stalls at cis-DCE (Figure 8a).

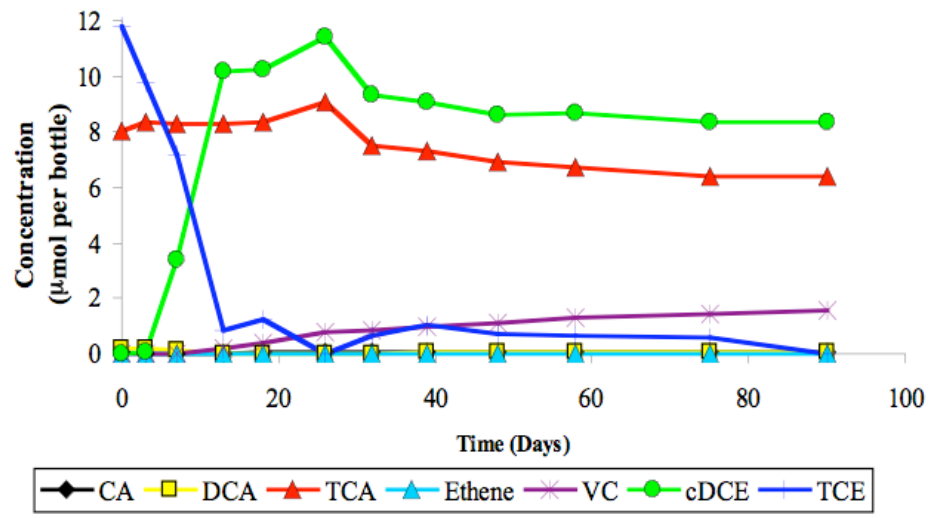

Figure 8a: Dechlorination of TCE only as far as cis-DCE in KB-1 cultures amended with both TCE and 1,1,1-TCA (30 mg/l each)

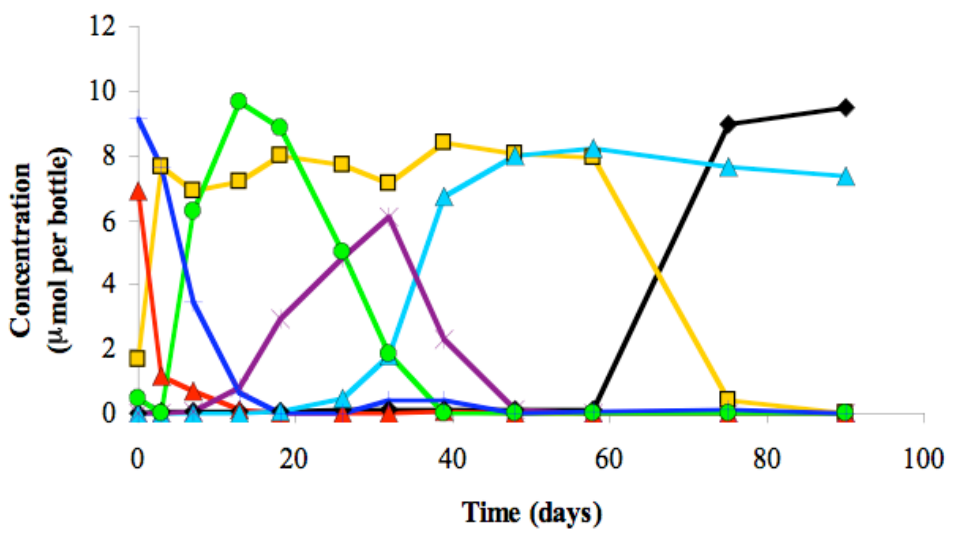

Figure 8b: Complete dechlorination of TCE and 1,1,1-TCA in a culture containing both KB-1 and a 1,1,1TCA dechlorinating enrichment culture. 
However, if KB-1 and the 1,1,1-TCA enrichment culture are mixed and amended with both TCE and 1,1,1-TCA, complete dechlorination of TCE to ethene and 1,1,1-TCA to chloroethane (CA) was observed, in a sequential manner (Figure 8b). These results suggest that inhibition of TCE dechlorination by 1,1,1-TCA is reversible, and can be avoided if active 1,1,1-TCA dechlorination can be stimulated (Grostern and Edwards, 2006b).

\subsection{Results for Phase 2 - Field samples}

Application of nucleic acid-based tools to field samples.

At all stages of tools development, we tested the 16S rRNA gene- and RDase gene-targeted approaches with groundwater samples collected from field sites undergoing enhanced treatment (i.e., biostimulation and bioaugmentation). These samples were provided by GeoSyntec, Regenesis and HSW Engineering. The validation of the DNA-based tools with field samples is ongoing, and current efforts focus on standardizing the procedures to ultimately develop a guidance document for analytical laboratories.

We had tentatively scheduled a visit from Dr. Chris Yeager from Savannah River National Laboratory (SRNL) to Dr. Löffler's laboratory at the Georgia Institute of Technology for 2006. The plans included training for Dr. Yeager in the qPCR approach and to analyze field samples from the Savannah River Site (SRS). Due to scheduling conflicts, these activities were postponed but we will try to reschedule or find alternative options to include SRS site samples in the analyses.

We successfully demonstrated that the application of RNA-targeted approaches to estimate general (i.e., 16S rRNA-targeted) and specific dechlorination (i.e., RDase gene-targeted) activity is feasible with groundwater samples. The RNA-targeted approach requires further development of procedures to assess biomarker RNA integrity during sampling, transport and storage and quantify RNA loss during biomass collection and

\subsection{SUMMARY AND FUTURE WORK}

This collaborative effort between Dr. E. Edwards at the University of Toronto and Dr. F. Löffler at the Georgia Institute of Technology developed new qualitative and quantitative tools for site assessment and bioremediation monitoring. The value and the limitations of Dehalococcoides $16 \mathrm{~S}$ rRNA gene-targeted approaches were evaluated and described. PCR approaches targeting specific RDase genes implicated in chlorinated ethene reductive dechlorination were designed and optimized for quantitative assessment. Application of these tools to groundwater samples demonstrated their applicability for site assessment and bioremediation monitoring. New dechlorinating isolates were obtained and chlorinated ethane-dechlorinating cultures were characterized.

Future efforts should focus on obtaining more enrichment cultures and isolates to comprehensively describe the diversity of chlorinated solvent-dechlorinating bacteria. A major shortcoming of the current tools is the limited knowledge of reductive dechlorination biomarker genes. The RDase gene microarray designed in this effort will provide a valuable platform to assign function to many more RDase genes, and design (RT)-qPCR tools to more comprehensively describe the reductively dechlorinating Dehalococcoides community.

\subsection{COLLABORATIVE EXCHANGES AND VISITS TO OTHER LABORATORIES}

\section{July 2004}

A project kickoff meeting via conference call was held on July 7th 2004 between Drs. Edwards (University of Toronto), Löffler (Georgia Tech), Bagwell (SRNL), and Brigmon (SRNL). Project funding became available in August (Georgia Tech) and September (University of Toronto). 


\section{November 2004}

Dr. Löffler and one of his students visited Dr. Edwards' laboratory at the University of Toronto for 2 days. The goals were to coordinate efforts, exchange expertise, plan future efforts, and enhance student interactions. Dr. Edwards' students working on the project gave presentations followed by intensive discussions, and we toured the microarray facilities at the University of Toronto. One outcome of these discussions was to begin to compile all the available information on reductive dehalogenase gene sequence, and to generate an Excel spreadsheet, in which to store existing sequences, and new sequences as they are discovered.

\section{March 2005:}

Dr. Ruth Richardson (Cornell University) and four students and postdocs from her group visited Dr. Elizabeth Edwards and her group at the University of Toronto on March 11-12. The purpose was to share ideas about quantitatively measuring RNA in cultures and inferring dechlorination rates. Dr. Richardson also obtained a sample of consortium KB-1 and growth medium in order to test a culture that metabolically dechlorinates VC to ethene, as compared to strain 195, which reduces VC cometabolically.

\section{June 2005: Annual ASM Meeting in Atlanta}

This opportunity was used to enhance interactions between students from the University of Toronto and Georgia Tech, and discuss and plan future experiments. Drs. Edwards (University of Toronto) and Löffler (Georgia Tech) also met with Drs. Brigmon (SRNL) and Yeager (SRNL) to discuss results and the current status of the project, and plan future efforts. A work plan was drafted.

\section{August 2005:}

August 9-11, 2005, Dr. Löffler (as keynote speaker) and Dr. Edwards (as a rapporteur) participated in the SERDP/ESTCP Molecular Biology Tools Workshop, in Charlottesville, Virginia. A report was prepared and is available at: http://docs.serdpestcp.org/viewfile.cfm?Doc=MBT\%20Workshop\%20Report\%2Epdf

\section{January 2006:}

Dr. Löffler spent one week in January, 2006, at the Technical University of Munich to explore the feasibility to apply FISH technology for the detection and quantification of Dehalococcoides. Initial experiments were promising and we will continue to explore this approach. Although qPCR will remain the quantification approach of choice, a second, PCR-independent procedure is desirable for validation of qPCR results.

\section{February 2006:}

Two of Dr. Edwards' students (A. Waller and C. Washer) attended a microarray training course offered by the University of Toronto Microarray Centre.

\section{March 2006:}

A student from Dr. Löffler's laboratory (Elizabeth Padilla) spent 4 days in Toronto learning optimized microarray protocols.

\section{May 2006:}

Together with Karen Vangelas from SRNL, Dr. Löffler organized a 2-day workshop at Georgia Tech with all principal and co-principal investigators funded through DOE-SRS program. In attendance were also Claire Sink who is with the Department of Energy (DOE) Headquarters in the Office of Cleanup Technologies and serves as the Project Manager for the MNA/EA project; Karen Adams who is the SRS Project Manager for the MNA/EA project; and Karen Vangelas who is with the Savannah River National Laboratory and is the Operations Lead for the MNA/EA project. Drs. Edwards and Löffler and one student from each group attended the Fifth International Conference on Remediation of Chlorinated and Recalcitrant Compounds in Monterrey, CA, to present research findings (two oral presentations and two poster presentations). 


\subsection{PAPERS PUBLISHED, IN PRESS, OR IN PREPARATION}

1. Dinglasan-Panlilio, Dworatzek, S., M. J., Mabury, S. A., and E.A. Edwards. 2006. Microbial oxidation of 1,2-dichlorethane under anoxic conditions with nitrate as electron acceptor in mixed and pure cultures. FEMS Microbiol. Ecol. 56.355-364.

2. Duhamel, M. and E.A. Edwards. 2006. Microbial composition of chlorinated ethene-degrading cultures dominated by Dehalococcoides. FEMS Microbiol. Ecol. 58: 538-549

3. Grostern, A., and E. A. Edwards. 2006a. Growth of Dehalobacter and Dehalococcoides spp. during degradation of chlorinated ethanes. Appl. Environ. Microbiol. 72:428-436.

4. Grostern, A. and E.A. Edwards. 2006b. A 1,1,1-trichloroethane-degrading anaerobic mixed culture enhances biotransformation of mixtures of chlorinated ethenes and ethanes. Appl. Environ. Microbiol. In press.

5. He, J., Y. Sung, R. Krajmalnik-Brown, K. M. Ritalahti, and F. E. Löffler. 2005. Isolation and characterization of Dehalococcoides sp. strain FL2, a trichloroethene (TCE)- and 1,2dichloroethene-respiring anaerobe. Environ. Microbiol. 7:1442-1450.

6. Krajmalnik-Brown, Y. Sung, R., K. M. Ritalahti, F. Michael Saunders, and F. E. Löffler. 2006. Environmental distribution of the trichloroethene reductive dehalogenase gene (tceA) suggests lateral gene transfer among Dehalococcoides. FEMS Microbiol. Ecol. In Press.

7. Löffler, F. E., and E. A. Edwards. 2006. Harnessing microbial activities for environmental cleanup. Current Opinion in Biotechnology. 17:274-284.

8. Löffler, F. E., R. A. Sanford, and K. M. Ritalahti. 2005. Enrichment, cultivation, and detection of reductively dechlorinating bacteria. Methods Enzymol. Vol. 397:77-111.

9. Ritalahti, K. M., B. K. Amos, Y. Sung, Q. Wu, S. S. Koenigsberg, and F. E. Löffler. 2006. Quantitative PCR targeting 16S rRNA and reductive dehalogenase genes simultaneously monitors multiple Dehalococcoides strains. Appl. Environ. Microbiol. 72:2765-2774.

10. Sung, Y., K. M. Ritalahti, R. P. Apkarian, and F. E. Löffler. 2006a. Quantitative PCR confirms purity of strain GT, a novel trichloroethene (TCE)-to-ethene respiring Dehalococcoides isolate. Appl. Environ. Microbiol. 72:1980-1987.

11. Sung, Y., K. E. Fletcher, K. M. Ritalahti, Ramos-Hernández, R. A. Sanford, N. M. Mesbah, and F. E. Löffler. 2006b. Geobacter lovleyi Strain SZ sp. nov., a novel metal-reducing and tetrachloroethene (PCE)-dechlorinating bacterium. Appl. Environ. Microbiol. 72:2775-2782.

12. Waller, A. S., R. Krajmalnik-Brown, F. E. Löffler, and E. A. Edwards. 2005. Multiple reductive-dehalogenase-homologous genes are simultaneously transcribed during dechlorination by Dehalococcoides-containing cultures. Appl. Environ. Microbiol. 71:8257-8264. 


\subsection{CONFERENCE PRESENTATIONS}

1. Löffler, F. E. Molecular Tools Supporting Efficient Bioremediation. Interstate Technology \& Regulatory Council (ITRC) Fall Meeting, October 23-27, Scottsdale, AZ.

2. Edwards, E.A. Genomes to Solutions - Bioremediation in the Genomics Era. Gordon Research Conference on Environmental Sciences: Water. June 25-28, 2006.

3. Löffler, F. E. 5/2006. Molecular Biological Tools to Support Bioremediation Applications: The Road Ahead, The 5th International Conference on Remediation of Chlorinated Compounds, Monterey, May 21-25, 2006, Monterey, CA.

4. Amos, B. K., E. J. Suchomel, K. D. Pennell, and F. E. Löffler. 2006. Microbial Distribution and Microbial Reductive Dechlorination Performance in the Immediate Vicinity of a NAPL Source Zone. Fifth International Conference on Remediation of Chlorinated and Recalcitrant Compounds. May 21-25, 2006, Monterey, CA.

5. Seguiti, F., C. Drummond, E. Petrovskis, F. E. Löffler, and K. M. Ritalahti. 2006. Management/site closure strategies for a trichloroethene (TCE) impacted aquifer following bioaugmentation. Fifth International Conference on Remediation of Chlorinated and Recalcitrant Compounds. May 21-25, 2006, Monterey, CA.

6. Waller, A. S., K. Mo, D. Zhang, C. Washer, B. Higgins, B. Curtis, S. Bowman, and E. A. Edwards. 2005. Microbial Community Genome Sequencing of Biodegrading Consortia. In Abstracts from the International Conference on Microbial Genomes; Halifax, Nova Scotia.

7. Waller A.S. K. Mo, C. Washer, and E.A. Edwards. 2005. Microarray Detection of Gene Expression During Dechlorination of a Dehalococcoides-Containing Mixed Culture - Presented at the 55th Canadian Chemical Engineering Conference, Toronto, ON, Canada. October 16-19.

8. Krajmalnik-Brown. R., F. M. Saunders, F. E. Löffler, K. M. Ritalahti. 2005. A Reductive Dehalogenase (RDase) Gene is Shared Among Dehalococcoides Species, abstr. R-039. American Society for Microbiology - General Meeting. June 5-9, Atlanta, Georgia.

9. Waller, A. S., K. Mo, D. Zhang, R. Krajmalnik-Brown, K. M. Ritalahti, F. E. Löffler, E. A. Edwards. 2005. Identification of Genes Involved in Anaerobic Dechlorination in a Dehalococcoides-Containing Mixed Consortium Using Microarray Analysis, abstr. Q-378. American Society for Microbiology - General Meeting. June 5-9, Atlanta, Georgia.

10. Sung, Y., K. M. Ritalahti, F. E. Löffler. 2005. Complete Detoxification of Trichloroethene (TCE) to Ethene by a New Dehalococcoides Species Designated Strain H10, abstr. Q-038. American Society for Microbiology - General Meeting. June 5-9, Atlanta, Georgia.

11. Grostern, A., E.A. Edwards. 2005. Investigation of Community Dynamics in Anaerobic Chlorinated Ethane-Degrading Microbial Cultures. American Society for Microbiology - ASM 105th General Meeting, June 5-7, Atlanta, GA

12. Duhamel, M., A. Waller, K. Mo, E.A. Edwards, P.C. Dennis, S. Dworatzek, X. Druar, E.R. Hendrickson, and D. Major. 2005. Importance of non-Dehalococcoides organisms in reductive dechlorinating cultures. Battelle In Situ and On-Site Bioremediation Symposium, June 6-9, Baltimore, MD.

Note that other federal funding agencies and industry sponsors, in addition to the DOE-SRS funding, contributed resources to support the research on reductive dechlorination in Drs. Edwards and Löffler's laboratories. However, the scope and objectives of the DOE-SRS funding had significant impact on the research productivity and direction. 


\subsection{RELATED PROPOSAL SUBMISSIONS}

A large Genome Canada grant application, entitled "Microbial Environmental Genome Alliance (MEGA)", to further pursue identification of metabolic and regulatory genes in dechlorinating cultures via genome analysis was submitted on January 28, 2005. This SRNL project (Drs. Edwards \& Löffler) and Dr. Ruth Richardson's SRNL project were included as co-funding for the Genome Canada grant application, which required 1:1 matching funds. This application solidified ongoing collaboration between Dr. Edwards and Ruth Richardson and Steve Zinder at Cornell University. The application made it through two stringent cuts, but was unfortunately eliminated in the final round of the selection process. The reviews, however, were very encouraging about the bioremediation metagenomic section of the application.

Dr. Edwards resubmitted a component of the Genome Canada application to Canada's National Science and Engineering Research Council (NSERC) and the application is pending.

A Proposal to the Joint Genome Institute (DOE-JGI) for community sequencing of the KB-1 culture was submitted March 2006 and was accepted June 2006.

In addition, Dehalococcoides strain GT will be sequenced by DOE-JGI; this complements genome sequences of Dehalococcoides strains 195, CBDB1, BAV1 and VS that are available or sequencing is in progress.

A proposal to SERDP entitled "BioReD: Biomarkers and Tools for Reductive Dechlorination Site Assessment, Monitoring, and Management" was submitted in March 2006. This proposal builds on the current results to expand the biomarker identification efforts, to develop a relative quantification approach and establish activity correlation factors to estimate in situ reductive dechlorination activity, and to perform field demonstration/validation. This proposal was successful and will enable the continuation of the work described herein. 